\title{
PREDICTION OF PATH LOSS ESTIMATE FOR A FREQUENCY MODULATION (FM) STATION USING BASIC TRANSMISSION LOSS MODEL
}

\author{
N. Y. GOSHWE, J. ORINYA AND G. A. IGWUE \\ (Received 23, May 2013; Revision Accepted 16, July 2013)
}

\begin{abstract}
This paper presents the path loss model that seeks to predict the signal strength degradation of Radio Nigeria, FM station, Makurdi which is normally a major component in the analysis and design of link budget of a telecommunication system. For the purpose of this work, Benue state was divided into four (4) routes for ease of data gathering, namely northern, southern, eastern and western parts and a Potomac FIM-41 field strength meter was used to measure the signal strength of the FM signal at selected locations. The values obtained are compared with those computed from Basic Transmission Loss Model for consistency. The result shows that values obtained from the model are slightly higher than the measured and it returned ninety-five percent (95\%) confidence interval for the two samples using degree of freedom of 22. A null hypothesis was applied and the measure of the level of significant difference between the model and measured values is $10.3 \%$. The results present no significant difference between the model and measured data. It can therefore be concluded that the model is valid and can be used to estimate path losses of FM signals in Benue State of Nigeria.
\end{abstract}

\subsection{INTRODUCTION}

Path loss (path attenuation) is the reduction in power density of an electromagnetic wave as it propagates through space. Path loss is a major component in the analysis and design of the link budget of a telecommunication system (Mike, 2007). Path loss may be due to many effects, such as free space loss, refraction, diffraction, reflection, aperture-medium coupling loss and absorption. It can also be influenced by terrain contours, environment (urban or rural, vegetation and foliage), propagation medium (dry or moist air), the distance between the transmitter and the receiver, and the height and location of antennas (Shalangwa, 2010 and Green, 2004).

This paper therefore aims at estimating path loss that causes signal strength degradation of Radio Nigeria Harvest FM station signals transmitted from Makurdi in Benue State. The FM band is chosen because majority of the radio stations being established in Nigeria operate in the FM band.

\subsection{Path Loss Models}

A number of path loss models have been developed and proposed for calculation of losses along a path. These models are valid for frequencies above 30 megahertz and 100 kilometres range (Sinclair, 2006 and ITU-R, 2005). Among the models available are Friis transmission equation and flat earth models, basic transmission loss, Longley-Rice, International telecommunication union (ITU) 567 and370 and Terrain-Integrated Rough Earth Model (TIREM).

For this application the basic transmission loss model is adopted because it captures the

N. Y. Goshwe, Department of Electrical and Electronics Engineering, University of Agriculture, Makurdi, Nigeria

J. Orinya, Department of Electrical and Electronics Engineering, University of Agriculture, Makurdi, Nigeria

G. A. Igwue, Department of Electrical and Electronics Engineering, University of Agriculture, Makurdi, Nigeria 
environmental losses, assumes a flat earth and does not require measuring the receiver antenna gains (Zamanillo, 2002). Other models are having inherent weaknesses and limitations in adopting them for this application. Among them are:

i. Friis Transmission model which weakness lies in the fact that it considers only the effect of free space spreading neglecting other effects in the transmission environment.

ii. The Long-Distance model is only suitable for indoor environment only and not valid for outdoor transmission.

iii. The Two Ray Ground Model has the advantage of accounting for reflections in the transmission channel and considers antenna height and gain which are critical in any radio link design, but for this work, it will be difficult to measure the antenna gain of end users across the state.

iv. The Terrain-Integrated Rough Earth Model (TIREM provides more accuracy in the radio propagation model than the free space path loss (FSPL) model because it takes into account the transmitting medium (surface refractivity and humidity), antenna properties (height, frequency and polarization), (conductivity and terrain elevations) but this research is limited in equipment to measure all the parameters.

v. The Longley-Rice model predicts only long-term median transmission loss over irregular terrain relative to free space transmission loss.

vi. The adjusted ITU-P1546 model is suitable only for modelling propagation path loss in the broadcasting, land mobile and certain fixed services (those employing point to multipoint systems in the frequency range $30-3000 \mathrm{MHZ}$ and for the distance range $1 \mathrm{KM}$ to $1000 \mathrm{KM}$ ).

\subsection{The Basic Transmission Loss Model}

This model describes the attenuation of radio waves under real condition and proposes a path loss model in terms of the attenuation function and attempts to resolve the design of radio links whose parameters are time invariant. The model specifically captures the free space path loss in addition to the effect of the transmission environment. Important factors like the wave length, power gains of the transmitting and receiving antenna are included in the expression. The model weakness lies in the fact that it assumes a flat earth which does not consider the effect of irregular terrain (hills and mountains) and diffraction (Green, 2004 and Jimenez, 1999). And it is worth noting that the terrain over which signals travel will have a significant effect on them especially the effect of multipath. This effect normally gives rise to fading which is neglected in the model.

This paper is adopting the basic transmission loss model because it captures the environment, frequency and distance which are important factors in path loss estimation. In addition, it does not require measuring the receiver antenna gains.

\subsection{The Fundamental Equation for Free Space Propagation}

The fundamental equation for free space transmission or propagation can be expressed using the average power $W_{T}$ assumed to be radiated in all directions (Isotropically) by a transmitting antenna (Salonen, 1992). It is further assumed that this isotropic radiator is placed in free space, a homogenous and non-absorbing medium of dielectric constant unity. In addition, it is assumed that this radiation will be spread out spherically as it travels away from the source which is expressed as

$$
W_{R}=W_{T} G_{T} G_{R}\left(\frac{\lambda}{4 \pi d}\right)^{2}
$$

$W_{T}$ is the Radiated power in watts, $W_{R}$ is the Received power in watts, $G_{T}$ is the maximum directivity gain of transmitting antenna, $G_{R}$ is the maximum directivity gain of receiving antenna, $\lambda$ is the wave length in metres and $d$ is the distance in metres.

The wavelength of the FM signal can be expressed as a function of frequency of signal using

$$
V=f \lambda
$$

Where $V$ is the velocity of light in free space $\left(3 \times 10^{8} \mathrm{~m} / \mathrm{s}\right), f$ is the signal frequency in hertz and $\lambda$ is the wavelength in metres

For an FM station transmitting at the frequency of $103.5 \mathrm{MHZ}$, the wavelength can be computed using equation (2) to obtain the value of 2.89 metre. This value is used to estimate the path losses for the selected locations and the values of the path loss were estimated as a function of the distance from Makurdi (the reference point).

The basic transmission loss model proposed a path loss model in terms of the attenuation function $F$ which can be expressed in terms of transmission loss as 
$L=\frac{1}{G_{1} G_{2}}\left(\frac{4 \pi r}{\lambda F}\right)$

If it is assumed that the receiving and transmitting antennas have unity gain, then Equation (3) can be reduced to equation (4) which is called the basic transmission loss equation.

$$
L_{b}=\left(\frac{4 \pi r}{\lambda F}\right)
$$

Equation (3) can also be expressed in decibel as $L=20 \log (4 \pi r)-20 \log \lambda-F(d B)-G_{1}(d B)-G_{2}(d B) \ldots(5)$ While equation (4) which is the basic transmission loss equation can be expressed in decibel as

$$
L_{b}=20 \log (4 \pi r)-20 \log \lambda-F(d B)
$$

Where $r$ is the distance between the transmitting and receiving antennas in metres, $\lambda$ is the wavelength of signal in metres, $G_{1}$ is the power gain of transmitting antenna, $G_{2}$ is the power gain of receiving antenna and $F$ is the attenuation function.

The basic transmission loss equation can be modified by substituting the attenuation function $F$ with the attenuation value in $\mathrm{dB}(20 \log \mathrm{A})$ and the distance covered from the radio station to be $d$. The modified Basic transmission Loss equation can be expressed as $L_{m b}=20 \log 4 \pi+20 \log \lambda-20 \log A \ldots$.

If we assume unity gain for the antennas, then equation (7) can be reduced to

$L_{m b}=61.97+20 \log d-20 \log \lambda$

Where $L_{m b}$ is the modified path loss equation in decibel, $d$ is the distance from transmitter to receiver in kilometres and $\lambda$ is the wave length of signal in metres

\subsection{Methodology}

In this work it is assumed that FM signals (88MHZ-108MHZ) are not affected by rain fall, so model covers both dry and raining season. Also, the areas under study have a regular terrain or flat surface with few scattered trees. Very high structures like cathedrals, mosque and skyscrapers are negligible. For the purpose of this research, Benue state was divided into four (4) routes for ease of data gathering, namely northern, southern, eastern and western parts. Makurdi where the transmitter is located was used as the reference point and other locations are as distributed on Table 1.0.

Table 1.0: Locations and their Various Distances with Reference to Makurdi (Transmitter Location)

\begin{tabular}{|l|l|l|l|l|}
\hline Sn & Location & Distance $(\mathrm{Km})$ & Bearing of locations & Direction \\
\hline 1 & Harvest Station & 0 & 0 & Reference \\
\hline 2 & Ikpayongo & 3.25 & $171 \quad 0^{\prime}$ & SE \\
\hline 3 & lgbor & 13.02 & $1662^{\prime}$ & SE \\
\hline 4 & Abinsi & 31.72 & $3931^{\prime}$ & NE \\
\hline 5 & Aliade & 32.56 & $191 \quad 4^{\prime}$ & SW \\
\hline 6 & Naka & 34.80 & $272 \quad 13^{\prime}$ & NW \\
\hline 7 & Annune & 34.93 & $85 \quad 38^{\prime}$ & NE \\
\hline 8 & Daudu & 41.87 & $844^{\prime}$ & NE \\
\hline 9 & Taraku & 43.71 & $221 \quad 17^{\prime}$ & SW \\
\hline 10 & Gboko & 51.12 & $116 \quad 11^{\prime}$ & SE \\
\hline 11 & Yandev & 52.18 & $11041^{\prime}$ & SE \\
\hline 12 & Otukpo & 58.02 & $223 \quad 10^{\prime}$ & SW \\
\hline
\end{tabular}

Potomac Fim-41 field strength meter was used to measure field strength of signal transmitted from the reference point to the selected locations. The universal traverse 
Mercator (geographical reference system) was used to calculate the line of sight distance from Harvest FM station (reference point) to locations under study. Line of sight distance, bearing (direction) of each town from the reference point was recorded and tabulated. In addition, the signal strength measured in volt was recorded against individual locations and the result tabulated. The power loss at each point from Makurdi (the reference point) was obtained using equation (9). The power loss in $\mathrm{db}$ is given as:

$$
\operatorname{Loss}(d B)=20 \log \left(\frac{V_{R}}{V_{S}}\right)
$$

$\mathrm{V}_{\mathrm{s}}$ is the Voltage (signal strength) at source

$V_{R}$ is the signal strength at a distance from the source

The two major data under this study are the road distance and field strength measured at the various locations. The distance along with other known and approximated parameters were used to estimate the path loss. Basic transmission model was used for modelling the path loss while MATLAB R2007b software was used in analysing the data. Statistical Package for social science (SPSS) was used to compare the data obtained from measured data and the model to test the reliability of the model in estimating the path loss.

\subsection{RESULTS AND DISCUSSION}

\subsection{Analysis of the Path Loss using the Field Strength Meter \\ MATLAB software was used to plot the} graph of path loss versus distance for measured data in Figure 1.0. There is a linear relationship between distance and path loss up to Ikpayongo $(13.2 \mathrm{Km})$ away from the reference point at 65 $\mathrm{dB}$. The relationship between path loss and distance for Abinsi, Igbor, Annune, Aliade, Taraku, Yandev, Gboko, Otukpo and Naka is non linear.

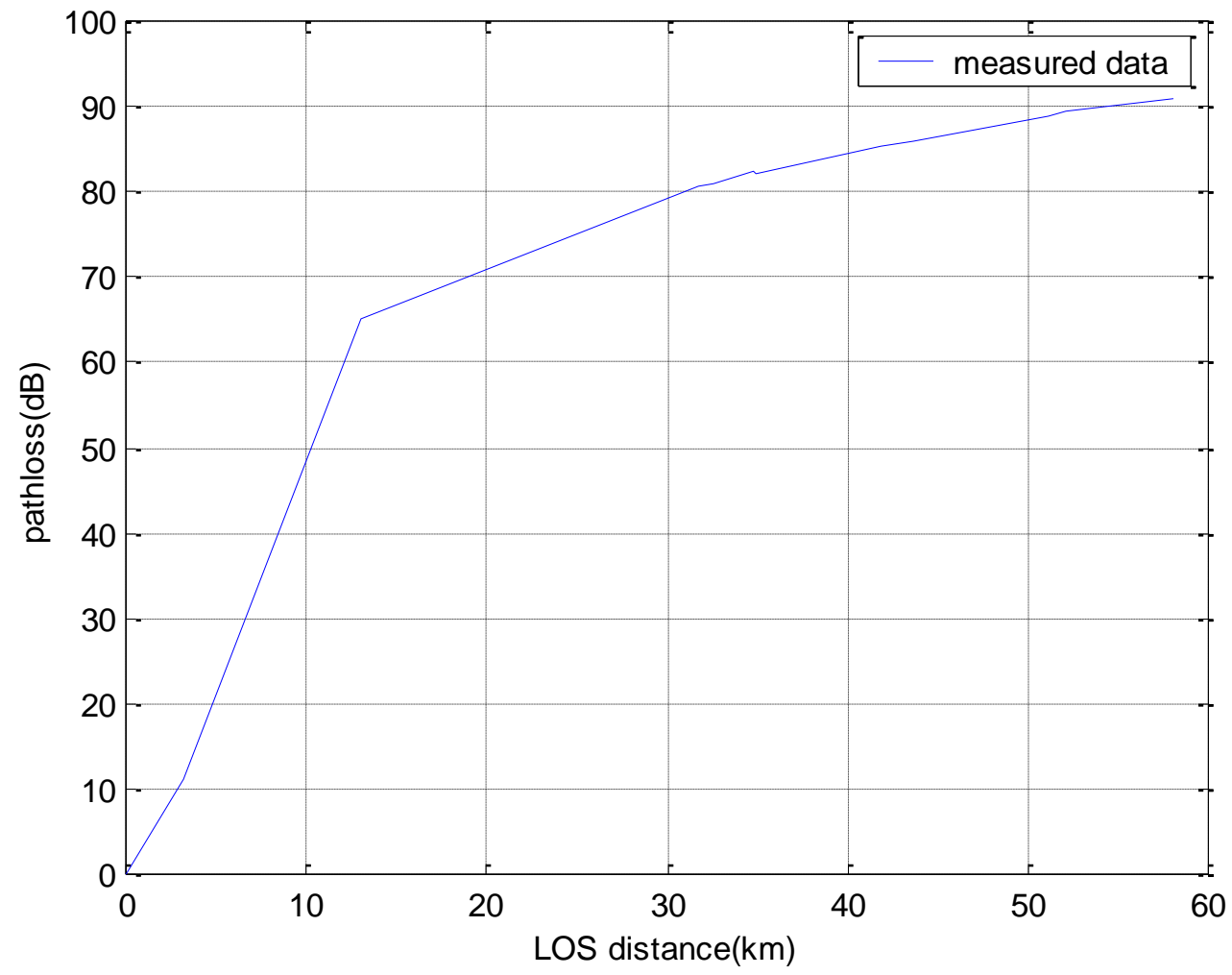

Figure 1.0: Graph of path loss versus distance for measured data 
The result shows that the ratio of power loss to distance decreases progressively as the distance increases from the transmitting point.
The T-test on the measured data gives the values on Table 2.0.

Table 2.0: One sample T-test: Measured data

\begin{tabular}{|l|l|l|l|l|l|}
\hline Variable & $\mathrm{N}$ & Mean & Standard deviation & Standard error mean & 95\% confidence interval \\
\hline Measured data & 11 & 76.65 & 22.78 & 6.87 & $61.34,91.95$ \\
\hline
\end{tabular}

\subsection{Analysis of Path Loss from the Model}

Simulation of the path loss using MATLAB gives the graph of path loss versus distance in Figure 2.0. There is a linear relationship between path loss and distance up to
Ikpayongo at a distance of $13.2 \mathrm{~km}$ with a loss of $62.99 \mathrm{~dB}$ almost similar to the measured values. The relationship between path loss and distance for other towns under study is nonlinear.

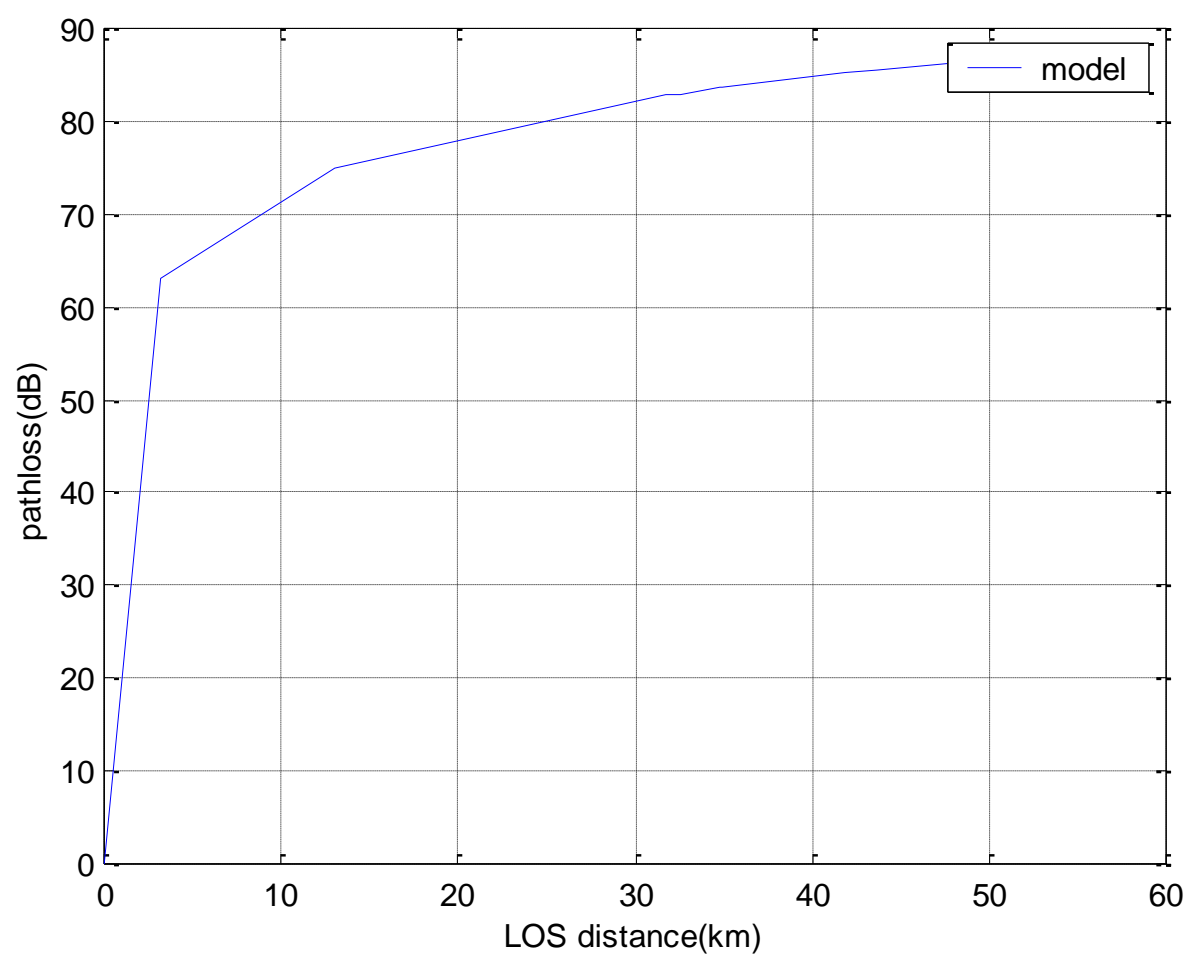

Figure 2.0: Graph of path loss versus distance for model

Analysis for consistency of the data from the model using statistical package (SPSS) produced the results shown in Table 3.0.

Table 3.0: One sample T-test: model

\begin{tabular}{|l|l|l|l|l|l|}
\hline Variable & $\mathrm{N}$ & Mean & $\begin{array}{l}\text { Standard } \\
\text { Deviation }\end{array}$ & Standard error mean & 95\% confidence interval \\
\hline Model & 11 & 82.16 & 7.24 & 2.18 & $77.29,87.03$ \\
\hline
\end{tabular}




\section{3}

Validation of the Model

The data obtained from the model is compared with those computed from measured data using the field strength meter. The result shows that values obtained from the model are slightly higher than the measured and it returned ninety-five percent (95\%) confidence interval for the two samples using degree of freedom of 22.

A null hypothesis was applied; assuming that Mean of model minus Mean of measured data is equal to zero. For a null hypothesis, probability value less than or equal to $5 \%$ means there is significant difference between the model and measured data. From the analysis of the two sample data, the measure of the level of significant difference between the model and measured values is $45.3 \%$. The result as presented on Table 4.0 shows that there is no significant difference between the model and measured data.

Table 4.0: Two sample T-test and confidence interval: model, measured data

\begin{tabular}{|l|l|l|l|l|}
\hline & $\mathrm{N}$ & Mean & Standard deviation & Standard error mean \\
\hline Model & 11 & 82.16 & 7.25 & 2.18 \\
\hline Measured data & 11 & 76.65 & 22.78 & 6.87 \\
\hline
\end{tabular}

From Figure 3.0, there is slight difference between measured and calculated power loss between distances of 3.25 and $13 \mathrm{~km}$ and the gap narrows as the distance increases. The results obtained from this analysis shows that the model is valid and can be used to estimate power loss of FM signals.

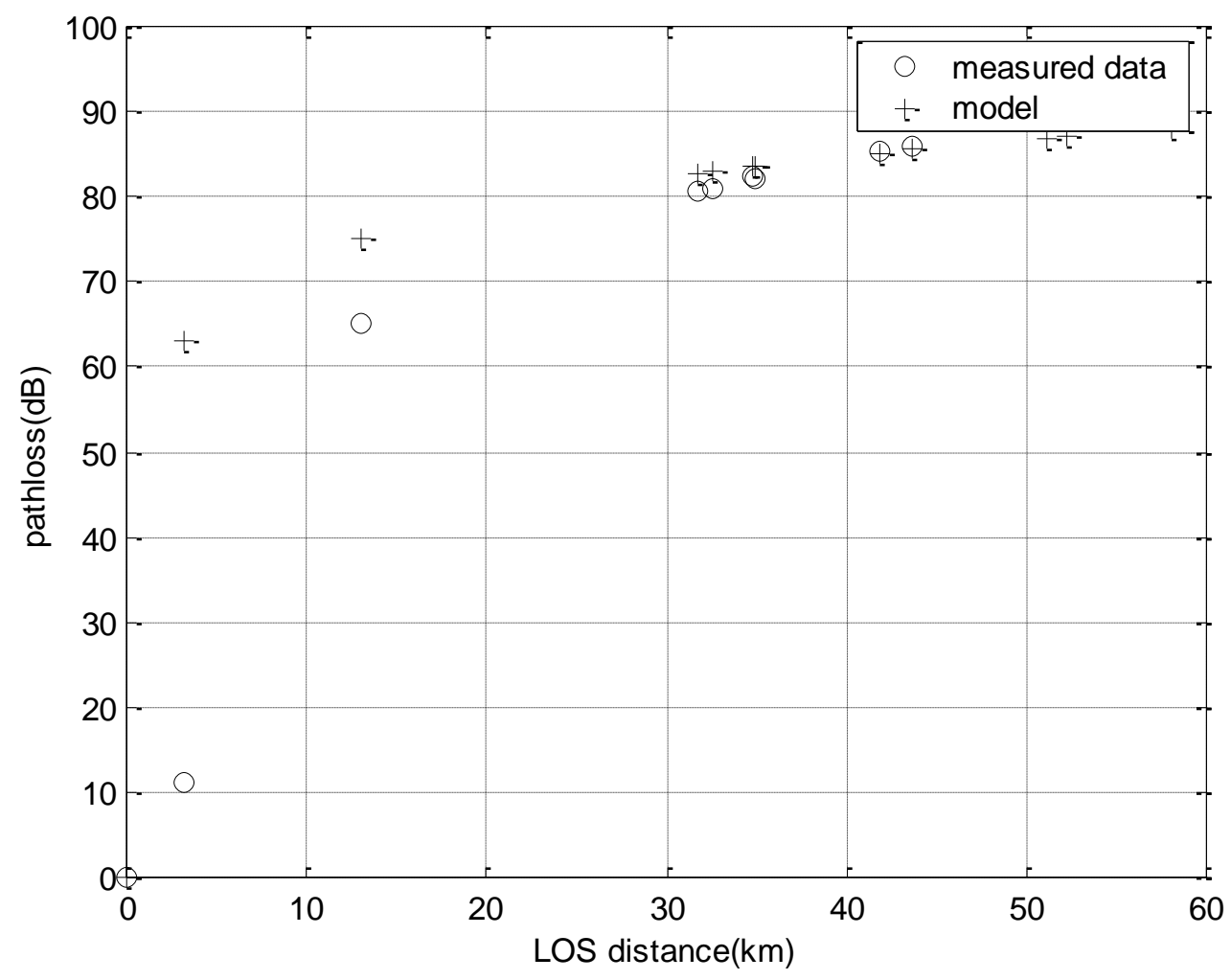

Figure 3.0: Graph of path loss versus distance for measured data and model 


\subsection{CONCLUSION}

The results shows that measured values are lower than values computed using the model. The relationship between path loss and distance for both the model and measured data are nonlinear. This should be expected because the model assumes ideal conditions and distances measured are approximations. It is worth noting that the condition under which radiated electromagnetic waves intensity measurement are performed may cause significant errors in results (Bartlett, 1992). From the analysis of the two sample data, there is no significant difference between the model and measured data. The model is therefore valid and can be used to estimate path losses of FM signals in Benue state. The information from this prediction can be a useful tool for link budget design for Radio Nigeria Harvest FM station and other FM stations in Makurdi. It will also provide a broad idea of path losses of FM signals to radio engineers operating in this environment.

\subsection{REFERENCES}

Bartlett, E., 1992. Electromagnetic Interference Measurement Methodologies' IEE Journal, 2, (6): 389-390.

Green, H., 2004. Propagation Impairment on Kaband Links in Tropical and Equatorial Regions. Antenna and Propagation Magazine. 46, (3): 36.

International telecommunication union (ITU-R)., 2005. Propagation Data and Prediction Methods required for the Design of Terrestrial Lineof-Sight Systems. Recommendations ITU-R 14, (3): 530-41.
Jimenez, F., 1999. A Practical Tropospheric Attenuation Model for Terrestrial Transmission. Proceedings of Fifth International Conference on Antennas and propagation, 2, (37): 273-276.

Mike, W., 2007. Introduction to Radio propagation for Fixed and Mobile Communication. Artech House Publishers. 105-109.

Salonen, E., 1992. Modelling and Calculation of Atmospheric Attenuation for Low Fade Margin Communications. ESA Journal, 16, (3): 299-317.

Shalangwa, D., 2010. Path Loss Propagation Model for Gombe Town Adamawa State Nigeria. International Journal of Computer Science and Network Security, 10, (6): 4.

Sinclair, J., 2006. World Wide Techniques for Predicting the Multipath Fading Distribution on Terrestrial LOS Links: Background and Result of Tests'. IEEE Transactions on Antenna and Propagation, 47, (1): 410-31.

Zamanillo, E., 2002. 'Path Loss Model for Broadcasting Applications and Outdoor Communication Systems in the VHF and UHF Bands' IEEE Trans. Broadcasting 48, (2): 91-96. 